\title{
A NEW APPROACH FOR BUCKLING ANALYSIS OF AXIALLY FUNCTIONALLY GRADED BEAMS
}

\author{
Jowita Rychlewska \\ Institute of Mathematics, Czestochowa University of Technology \\ Czestochowa, Poland \\ jowita.rychlewska@im.pcz.pl
}

\begin{abstract}
The object of considerations are axially functionally graded (FG) beams, which are loaded by an axial force varying along the length of the beam. The main idea presented here is to approximate FG beams by an equivalent beam with piecewise exponentially varying material properties, geometrical properties and axial load. Numerical solutions of the buckling analysis are obtained for four various types of boundary conditions associated with pinned and clamped ends. The usefulness of the proposed method is confirmed by comparing numerical results with those available for graded beams of special polynomial non-homogeneity.
\end{abstract}

Keywords: functionally graded beams, buckling load, Euler-Bernoulli beam theory

\section{Introduction}

Functionally graded materials represent a class of composites that have a gradually varying of material and/or geometrical properties in the specified direction. A review of researches on functionally graded materials can be found in Suresh and Mortensen [1]. In the present contribution the object of investigations are functionally graded (FG) beams. The gradient variation in FG beams may be oriented along the cross-section and/or axial direction.

A list of papers on buckling behaviour of FG beams with thickness-wise gradient variation is very extensive. For example, in paper [2] by Vo et al. the finite element model for vibration and buckling of functionally graded sandwich beams, based on a refined shear deformation theory, is presented. Li and Batra [3] derived analytical relations between the critical buckling load of a functionally graded Timoshenko beam and that of the corresponding homogeneous Euler-Bernoulli beam subjected to axial compressive load. Free vibrations, buckling and post-buckling of functionally graded beams containing open cracks, by assuming an exponential variation of material properties in the thickness direction, were studied by Yang and Chen [4]. 
For axially graded beams, stability problems are becoming more complicated because of the governing equation with variable coefficients. For example, Singh and $\mathrm{Li}$ [5] investigated the stability of axially functionally graded tapered beams through discretising a non-uniform column into a stepped multi-uniform column and solving a transcendental equation to compute the critical buckling load. Free vibrations and stability of axially functionally graded tapered Euler-Bernoulli beams were studied by Shahba and Rajasekaran [6] by using the differential transform element method. The finite element approach to the free vibration and stability analysis of axially functionally graded tapered Timoshenko beams was applied by Shahba et al. [7]. A new approach to exactly calculate the critical buckling loads of beams with arbitrarily axial inhomogeneity was presented by Huang and Luo in [8].

In this paper the stability analysis of axially graded beams with a distributed axial load is made. Considerations are carried out in the framework of the Euler-Bernoulli beam theory. It is assumed that the changes of material properties as well as the axial load are approximated by an exponential form. The obtained solutions of the buckling analysis for clamped-clamped, pinned-pinned, clamped-pinned and pinned-clamped beams are applied for numerical computations. Critical buckling loads are determined from the existence condition of a non-trivial solution in the system of algebraic equations obtained here. The proposed approach is based on these presented by Kukla and Rychlewska in [9] and Rychlewska in [10].

\section{Formulation of the problem}

In this paper an axially graded and non-uniform beam of length $L$ (along the $x$ direction) is considered. According to the Euler-Bernoulli beam theory, the governing differential equation for free transverse displacement $w(x)$ of axially FG beams loaded by an axial force $P(x)$ is given by

$$
\frac{d^{2}}{d x^{2}}\left[E(x) I(x) \frac{d^{2} w}{d x^{2}}\right]+\frac{d}{d x}\left[P(x) \frac{d w}{d x}\right]=0, \quad 0<x<L,
$$

where $I(x)$ is the moment of inertia and $E(x)$ denotes modulus of elasticity. Introducing the non-dimensional variable $\xi=x / L$, we rewrite equation (1) in the following form

$$
\frac{d^{2}}{d \xi^{2}}\left[E(\xi) I(\xi) \frac{d^{2} w}{d \xi^{2}}\right]+\frac{d}{d \xi}\left[P(\xi) \frac{d w}{d \xi}\right]=0, \quad 0<\xi<1
$$

where we still denote $E(x), I(x), P(x)$ and $w(x)$ as $E(\xi), I(\xi), P(\xi)$ and $w(\xi)$, respectively. Next, we assume that 


$$
E(\xi) I(\xi)=D_{0} g(\xi) \quad P(\xi)=P_{0} h(\xi), \quad 0<\xi<1,
$$

where $D_{0}=E(0) I(0)$ and $P_{0}=P(0)$. In this contribution we shall approximate functions $g(\cdot)$ and $h(\cdot)$ by piecewise exponentially functions, setting

$$
\begin{aligned}
& g(\xi) \cong D_{i} e^{2 \beta_{i} \xi} \\
& h(\xi) \cong P_{i} e^{2 \beta_{i} \xi}
\end{aligned} \quad \xi_{i-1}<\xi<\xi_{i}, \quad i=1, \ldots, n
$$

where $\xi_{0}=0$ and $\xi_{n}=1$. In order to determine coefficients $D_{i}, P_{i}, \beta_{i}, i=1, \ldots, n$, we assume that

$$
\begin{aligned}
& g\left(\xi_{i-1}\right)=D_{i} e^{2 \beta_{i} \xi_{i-1}} \\
& g\left(\xi_{i}\right)=D_{i} e^{2 \beta_{i} \xi_{i}} \\
& h\left(\frac{\xi_{i}+\xi_{i-1}}{2}\right)=P_{i} e^{\beta_{i}\left(\xi_{i}+\xi_{i-1}\right)}
\end{aligned} \quad i=1, \ldots, n
$$

Hence, we obtain

$$
\begin{aligned}
& \beta_{i}=\frac{1}{2\left(\xi_{i}-\xi_{i-1}\right)} \ln \frac{g\left(\xi_{i}\right)}{g\left(\xi_{i-1}\right)} \\
& D_{i}=g\left(\xi_{i}\right) e^{-2 \beta_{i} \xi_{i}} \quad i=1, \ldots, n \\
& P_{i}=h\left(\frac{\xi_{i}+\xi_{i-1}}{2}\right) e^{-\beta_{i}\left(\xi_{i}+\xi_{i-1}\right)}
\end{aligned}
$$

Assuming

$$
w(\xi)=w_{i}(\xi) \quad \xi_{i-1}<\xi<\xi_{i}, \quad i=1, \ldots, n
$$

and introducing denotations

$$
\lambda=\frac{P_{1} L^{3}}{D_{1}}, \quad \mu_{i}=\frac{P_{i} D_{1}}{D_{i} P_{1}} \quad i=1, \ldots, n
$$

the governing equation for the $i$-th segment of the beam can be written in the form

$$
\frac{d^{2}}{d \xi^{2}}\left[e^{2 \beta_{i} \xi} \frac{d^{2} w_{i}}{d \xi^{2}}\right]+\lambda \mu_{i} \frac{d}{d \xi}\left[e^{2 \beta_{i} \xi} \frac{d w_{i}}{d \xi}\right]=0, \quad \xi_{i-1}<\xi<\xi_{i}, \quad i=1, \ldots, n
$$

After some transformations equations (9) can be rewritten as 


$$
\frac{d^{4} w_{i}}{d \xi^{4}}+4 \beta_{i} \frac{d^{3} w_{i}}{d \xi^{3}}+\left(\lambda \mu_{i}+4 \beta_{i}^{2}\right) \frac{d^{2} w_{i}}{d \xi^{2}}+2 \beta_{i} \lambda \mu_{i} \frac{d w_{i}}{d \xi}=0, \quad \xi_{i-1}<\xi<\xi_{i}, \quad i=1, \ldots, n
$$

Equations (10) are completed by boundary and continuity conditions. The continuity conditions have the form

$$
\begin{array}{ll}
w_{i}\left(\xi_{i}\right)=w_{i+1}\left(\xi_{i}\right) & \frac{d w_{i}}{d \xi}\left(\xi_{i}\right)=\frac{d w_{i+1}}{d \xi}\left(\xi_{i}\right) \\
\frac{d^{2} w_{i}}{d \xi^{2}}\left(\xi_{i}\right)=\frac{d^{2} w_{i+1}}{d \xi^{2}}\left(\xi_{i}\right) & \frac{d^{3} w_{i}}{d \xi^{3}}\left(\xi_{i}\right)=\frac{d^{3} w_{i+1}}{d \xi^{3}}\left(\xi_{i}\right), \quad i=1, \ldots, n-1
\end{array}
$$

In this contribution we consider the following four types of boundary conditions (BC):

- clamped-clamped beam (C-C)

$$
w_{1}(0)=0, \quad \frac{d w_{1}}{d \xi}(0)=0, w_{n}(1)=0, \quad \frac{d w_{n}}{d \xi}(1)=0
$$

- pinned-pinned beam (P-P)

$$
w_{1}(0)=0, \quad \frac{d^{2} w_{1}}{d \xi^{2}}(0)=0, \quad w_{n}(1)=0, \quad \frac{d^{2} w_{n}}{d \xi^{2}}(1)=0
$$

- clamped-pinned beam (C-P)

$$
w_{1}(0)=0, \quad \frac{d w_{1}}{d \xi}(0)=0, w_{n}(1)=0, \quad \frac{d^{2} w_{n}}{d \xi^{2}}(1)=0
$$

- pinned-clamped beam (P-C)

$$
w_{1}(0)=0, \quad \frac{d^{2} w_{1}}{d \xi^{2}}(0)=0, \quad w_{n}(1)=0, \quad \frac{d w_{n}}{d \xi}(1)=0
$$

\section{A solution to the problem}

In this section a solution of the buckling problem (10)-(11) $+\mathrm{BC}$ is discussed. Under assumption that: $\beta_{i}^{2}<\lambda \mu_{i}, i=1, \ldots, n$, the general solution of equations (10) has the following form

$$
w_{i}(\xi)=C_{1 i}+C_{2 i} e^{-2 \beta_{i} \xi}+C_{3 i} e^{-\beta_{i} \xi} \cos \left(\delta_{i} \xi\right)+C_{4 i} e^{-\beta_{i} \xi} \sin \left(\delta_{i} \xi\right), \quad i=1, \ldots, n
$$


where $\delta_{i}=\sqrt{\lambda \mu_{i}-\beta_{i}^{2}}, C_{1 i}, C_{2 i}, C_{3 i}, C_{4 i} \in R, i=1, \ldots, n$. Substituting functions (16) into one of the set of boundary conditions (12)-(15) and continuity conditions (11), we obtain a homogeneous system of $4 n$ linear equations with respect to the unknowns $C_{1 i}, C_{2 i}, C_{3 i}, C_{4 i} \in R, i=1, \ldots, n$. This system of equations can be written in the matrix form

$$
\mathbf{A}(\lambda) \cdot \mathbf{X}=\mathbf{0}
$$

where $\mathbf{X}=\left[C_{11}, C_{21}, C_{31}, C_{41}, \ldots, C_{1 n}, C_{2 n}, C_{3 n}, C_{4 n}\right]^{T}$ and $\mathbf{A}(\lambda)=\left[a_{k j}\right]_{4 n \times 4 n}$. Two first rows of the matrix $\mathbf{A}$ represent the boundary conditions at $\xi=0$ and the two last rows of $\mathbf{A}$ represent the boundary conditions at $\xi=1$. The rows of the matrix $\mathbf{A}$ with non-zero elements $a_{4 i-1,4 i-3}, \ldots, a_{4 i-1,4 i+4}, a_{4 i, 4 i-3}, \ldots, a_{4 i, 4 i+4}, a_{4 i+1,4 i-3}, \ldots$, $a_{4 i+1,4 i+4}, a_{4 i+2,4 i-3}, \ldots, a_{4 i+2,4 i+4}, i=1, \ldots, n-1$, are determined by the continuity conditions at $\xi=\xi_{i}, i=1, \ldots, n-1$. For the existence of a non-trivial solution to the buckling load problem, it is necessary that the determinant of the matrix $\mathbf{A}$ has to be equal to zero

$$
\operatorname{det} \mathbf{A}(\lambda)=0
$$

In the subsequent section equation (18) is solved numerically with respect to dimensionless critical buckling load $\lambda$, using an approximate method.

\section{Numerical computations}

In this section some numerical results are presented. The numerical computations were carried out for an FG beam with $n$ segments of the same length. Non-dimensional critical buckling loads for an FG beam with functions $g(\xi)=(1+\xi)^{2}$ and $h(\xi)=1$ obtained in the present study for $n=75$ are listed in Table 1 in comparison with those presented in [8] by Huang and Luo. It can be seen that the present results are in good agreement with these given in the paper [8].

Table 1

Non-dimensional critical buckling loads of a beam with $g(\xi)=(1+\xi)^{2}, h(\xi)=1$

\begin{tabular}{|l|c|c|c|}
\hline \multicolumn{1}{|c|}{$\begin{array}{c}\text { Boundary } \\
\text { conditions }\end{array}$} & C-C & C-P & P-C \\
\hline Huang and Luo [8] & 81.9233636440 & 42.1091761225 & 42.1091761216 \\
\hline Present results & 81.9573051028 & 42.0202358701. & 42.0196941905. \\
\hline
\end{tabular}


Next, we take into account functions $g(\xi)=(1+\gamma \xi)^{2}$ and $h(\xi)=1+0.5 \sin (\eta \pi \xi)$ for $\gamma=-0.5 ; 0.5$ and $\eta=1 ; 2$. The effects of parameters $\gamma, \eta$ on the non-dimensional critical buckling load for different boundary conditions are presented in Table 2 . The computations have been provided assuming numbers of beam segments: $n=5$; $n=10 ; n=20$.

Table 2

Non-dimensional critical buckling loads of a beam for different boundary conditions, $g(\xi)=(1+\gamma \xi)^{2}, h(\xi)=1+0.5 \sin (\eta \pi \xi)$

\begin{tabular}{|c|c|c|c|c|c|}
\hline $\mathrm{BC}$ & $\eta$ & $\gamma$ & $n=5$ & $n=10$ & $n=20$ \\
\hline \multirow{4}{*}{$\mathrm{C}-\mathrm{C}$} & \multirow{2}{*}{1} & -0.5 & 18.0326618862 & 16.000812014 & 15.037147626 \\
\hline & & 0.5 & 42.3467952861 & 41.3814908015 & 40.8418684385 \\
\hline & \multirow{2}{*}{2} & -0.5 & 30.9167717114 & 25.9579817435 & 23.5869092065 \\
\hline & & 0.5 & 67.315838847 & 63.6530330003 & 60.9822288428 \\
\hline \multirow{4}{*}{ P-P } & \multirow{2}{*}{1} & -0.5 & 4.44181720275 & 3.94580897007 & 3.70934657965 \\
\hline & & 0.5 & 10.9508717389 & 10.7004056583 & 10.561963588 \\
\hline & \multirow{2}{*}{2} & -0.5 & 6.91670862246 & 5.84577734899 & 5.31755856644 \\
\hline & & 0.5 & 16.854852943 & 15.7864981399 & 15.1113705843 \\
\hline \multirow{4}{*}{$\mathrm{C}-\mathrm{P}$} & \multirow{2}{*}{1} & -0.5 & 9.53744854968 & 8.4654491907 & 7.9560423312 \\
\hline & & 0.5 & 22.4785604313 & 21.9789821551 & 21.6978091336 \\
\hline & \multirow{2}{*}{2} & -0.5 & 20.6655195425 & 17.8293864056 & 16.2839659863 \\
\hline & & 0.5 & 49.61145296 & 48.101957009 & 46.4246271002 \\
\hline \multirow{4}{*}{$\mathrm{P}-\mathrm{C}$} & \multirow{2}{*}{1} & -0.5 & 9.34726465564 & 8.30242812168 & 7.80486693848 \\
\hline & & 0.5 & 22.7660844119 & 22.229522437 & 21.9376428651 \\
\hline & \multirow{2}{*}{2} & -0.5 & 11.8648160278 & 9.92227510151 & 9.00715765748 \\
\hline & & 0.5 & 27.9845620671 & 25.945953544 & 24.7770408754 \\
\hline
\end{tabular}

The non-dimensional critical buckling loads for functions $g(\xi)=(1+\gamma \xi)^{2}$, $h(\xi)=(1+\gamma \xi)^{\alpha}$ for $\alpha=1 ; 1.5 ; 2 ; 2.5 ; 3$ and $\gamma \in(0,1)$ are presented in Figure 1. The calculations were performed for four types of boundary conditions (12)-(15). It can be noticed that variation of parameter $\gamma$ has signification effect on the critical buckling load.

For $\gamma \rightarrow 0$ we obtain the case of a homogeneous beam subjected to a constant load. In this case the critical buckling load can be exactly calculated and they have the following values [11]:

(1) $\lambda=39.4784$ for clamped-clamped beam;

(2) $\lambda=9.8696$ for pinned-pinned beam;

(3) $\lambda=20.1907$ for clamped-pinned or pinned-clamped beam. 
From Figure 1, it is seen that the obtained results for $\gamma \rightarrow 0$ agree well with these presented above.
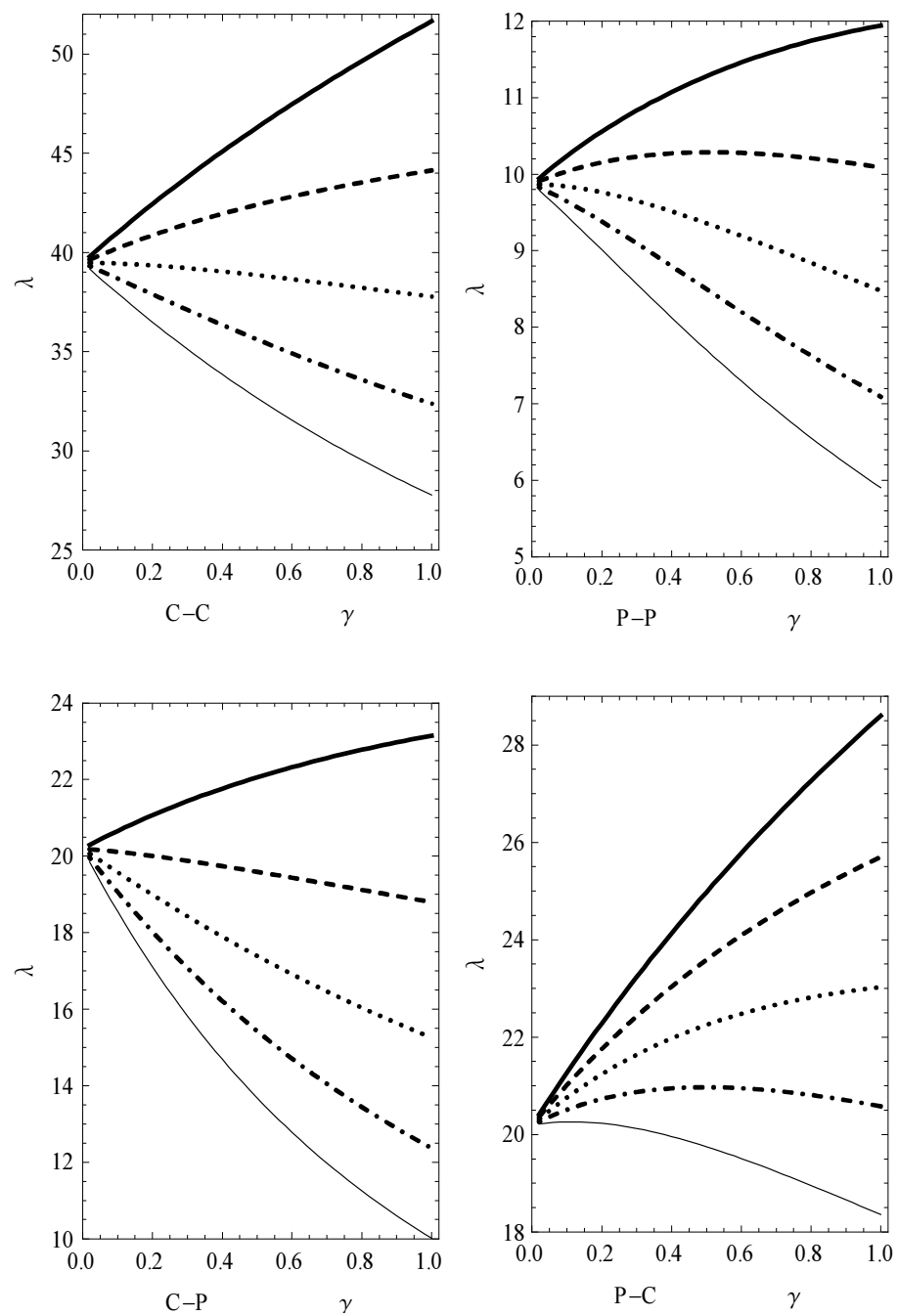

Fig. 1. Non-dimensional critical buckling loads with $g(\xi)=(1+\gamma \xi)^{2}, h(\xi)=(1+\gamma \xi)^{\alpha}$ as a function of $\gamma$ for $\alpha=1$ (black bold line), $\alpha=1.5$ (dashed line), $\alpha=2$ (dotted line), $\alpha=2.5$ (dotted-dashed line), $\alpha=3$ (black solid line) for different boundary conditions

\section{Conclusions}

In this paper a new method, which is capable of computing the buckling loads of functionally graded beams in the axial direction subjected to a distributed axial load, is presented. The beams under consideration are approximated by another 
beam with piecewise exponentially varying both geometrical/material properties and axial load. The proposed method has been verified with the previous results and has found good agreement with them. Numerical results are presented in both tabular and graphical forms to show the influence of different material distribution, load distribution and boundary conditions on the critical buckling loads of FG beams. The effect of these parameters on the buckling behaviour is significant.

\section{References}

[1] Suresh S., Mortensen A., Fundamentals of Functionally Graded Materials, The University Press, Cambridge 1998.

[2] Vo T.P., Thai H-T., Nguyen T-K., Maheri A., Lee J., Finite element model for vibration and buckling of functionally graded sandwich beams based on a refined shear deformation theory, Engineering Structures 2014, 64, 12-22.

[3] Li S-R., Batra R.C., Relations between buckling loads of functionally graded Timoshenko and homogeneous Euler-Bernoulli beams, Composite Structures 2013, 95, 5-9.

[4] Yang J., Chen Y., Free vibration and buckling analyses of functionally graded beams with edge cracks, Composite Structures 2008, 83, 48-60.

[5] Singh K.V., Li G., Buckling of functionally graded and elastically restrained non-uniform columns, Composites B 2009, 40, 393-403.

[6] Shahba A, Rajasekaran S., Free vibration and stability of tapered Euler-Bernoulli beams made of axially functionally graded materials, Appl. Math. Model. 2012, 36, 3094-3111.

[7] Shahba A., Attarnejad R., Tavanaie Marvi M., Hajilar S., Free vibration and stability analysis of axially functionally graded tapered Timoshenko beams with classical and non-classical boundary conditions, Composites B 2011, 42, 801-808.

[8] Huang Y., Luo Q.Z., A simple method to determine the critical buckling loads for axially inhomogeneous beams with elastic restraint, Comput. Math. Appl. 2011, 61, 2510-2517.

[9] Kukla S., Rychlewska J., Free vibration of axially functionally graded Euler-Bernoulli beams, Journal of Applied Mathematics and Computational Mechanics 2014, 13(1), 39-44.

[10] Rychlewska J., Buckling analysis of axially functionally graded beams, Journal of Applied Mathematics and Computational Mechanics 2014, 13(4), 103-108.

[11] Wang C.M., Wang C.Y., Reddy J.N., Exact Solutions for Buckling of Structural Members, CRC Press, LLC, Florida 2005. 